\title{
Nefrectomía laparoscópica: experiencia en el Centro Médico ABC, México
}

\section{Laparoscopic nephrectomy: experience in the ABC Medical Center, Mexico}

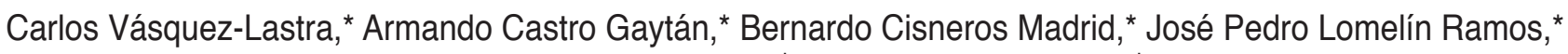 \\ Johnatan Vitar Sandoval, ${ }^{\star}$ Arturo Contreras Escamilla, ${ }^{\ddagger}$ Carla Osornio Vázquez ${ }^{\ddagger}$
}

Citar como: Vásquez-Lastra C, Castro GA, Cisneros MB, Lomelín RJP, Vitar SJ, Contreras EA et al. Nefrectomía laparoscópica: experiencia en el Centro Médico ABC, México. An Med (Mex). 2021; 66 (1): 36-41. https://dx.doi.org/10.35366/99487

\section{RESUMEN}

Introducción: La nefrectomía laparoscópica es hoy en día el procedimiento de elección en casos seleccionados comparada con la cirugía abierta gracias a su baja morbilidad, mortalidad y rápida reintegración a las actividades normales del paciente. Objetivo: Presentar nuestra experiencia en nefrectomía laparoscópica tanto de patología oncológica como benigna, abordando características trans- y postoperatorias, así como criterios de selección para la población en cuestión. Material y métodos: De enero del 2006 a enero del 2020, un total de 110 pacientes fueron tratados con nefrectomía laparoscópica por nuestro grupo. Se documentó tiempo quirúrgico, sangrado, días de estancia intrahospitalaria, complicaciones, así como estirpe histológico definitivo. Resultados: El tiempo quirúrgico promedio fue de 131 minutos. De acuerdo con la indicación quirúrgica, 67 pacientes $(61 \%)$ fueron sometidos a nefrectomía radical, $22(20 \%)$ a nefroureterectomía y 21 pacientes $(19 \%)$ a nefrectomía simple por exclusión renal. El tamaño tumoral promedio fue de $6.4 \mathrm{~cm}$ (rango 2.7 a $12 \mathrm{~cm}$ ). El sangrado transoperatorio promedio fue de $103 \mathrm{~mL}$ (rango de 10-2,000 $\mathrm{mL}$ ) y el promedio de días de estancia intrahospitalaria fue de 2.7 días. Sólo se presentó un caso de sangrado postoperatorio que se resolvió mediante laparoscopia. Conclusiones: La cirugía laparoscópica se ha establecido como el estándar de oro para el manejo de cáncer renal. Es también el procedimiento de elección en nefroureterectomía y en nefrectomía simple, así como en procedimientos reconstructivos de vías urinarias. Los resultados estéticos, con una rápida recuperación y

\section{ABSTRACT}

Introduction: Laparoscopic nephrectomy is currently considered as the preferred option over open surgery in select cases due to its low morbidity, risk and overall patient satisfaction. Objective: To present our experience in benign and oncologic laparoscopic nephrectomy, analyzing operative and postoperative characteristics of the population studied. Material and methods: From January 2006 to January 2020 a total of 110 patients were treated with laparoscopic nephrectomy. Surgical time, intraoperative bleeding, hospital stay and complications were documented. Results: Radical nephrectomy was performed in 67 (61\%) patients, nephroureterectomy in 22 (20\%) and simple nephrectomy in 21 (19\%). Tumor size was $6.4 \mathrm{~cm}$ (range 2.7-12 cm). Bleeding was $103 \mathrm{~mL}$ (range 10-2,000 $\mathrm{mL}$ ) and length of stay was 2.7 days. Only one case of reintervention was recorded due to postoperative bleeding that was resolved laparoscopically. Conclusions: Renal laparoscopic surgery is currently the gold standard for localized renal tumors, urothelial carcinomas, simple nephrectomies and reconstructive procedures. Cosmetic results along with a fast recovery and good oncologic control make it the procedure of choice for kidney surgery.

\footnotetext{
* Urólogo.

‡ Médico General.
}

Departamento de Urología, Centro Médico ABC. Ciudad de México, México.

Recibido: 30/06/2020. Aceptado: 15/03/2021.
Correspondencia: Dr. Carlos Vásquez-Lastra

Hospital ABC Campus Observatorio.

Sur 136 Torre Central, 40 piso, consultorio 8,

Col. Las Américas, Álvaro Obregón,

01120, CDMX.

E-mail: drcvlastra@hotmail.com 
reincorporación a actividades laborales, en conjunto con un adecuado control oncológico, la hacen el procedimiento de elección en patología renal.

Palabras clave: Cirugía laparoscópica, cáncer renal, nefrectomía, nefrectomía laparoscópica.

Nivel de evidencia: IV
Keywords: Laparoscopic surgery, renal cancer, nephrectomy, laparoscopic nephrectomy.

Level of evidence: IV

\section{INTRODUCCIÓN}

A partir de la primera nefrectomía laparoscópica realizada por Clayman en $1990,{ }^{1}$ el interés hacia este abordaje para cirugía renal ha crecido de manera exponencial. ${ }^{2,3}$ La cirugía renal por laparoscopia es ya una realidad en la comunidad urológica ${ }^{4,5}$ y sus indicaciones se han extendido de nefrectomía simple por enfermedad benigna a nefrectomía radical por enfermedad maligna. ${ }^{6}$ De igual manera, se ha incrementado la capacidad de realizar nefrectomías en tumores de mayor tamaño, nefrectomías parciales y nefroureterectomías. ${ }^{7}$ La cirugía robótica se ha establecido como una excelente herramienta del cirujano laparoscopista, la cual ha ayudado a realizar casos cada vez más complejos. Sin embargo, la cirugía renal sigue siendo un área donde la cirugía laparoscópica convencional tiene los mismos resultados que la cirugía robótica en casos concretos, ${ }^{8,9}$ a una fracción del costo.

Las ventajas del abordaje laparoscópico, en comparación con la cirugía abierta tradicional, se han descrito como: menor dolor postoperatorio, requerimiento de analgésicos ${ }^{10,11}$ y sangrado, ${ }^{12}$ reincorporación rápida a la actividad laboral y menores complicaciones, así como un mejor resultado cosmético. Múltiples estudios han demostrado que el resultado oncológico con abordaje laparoscópico es comparable con la cirugía abierta. ${ }^{13,14}$ La progresión tumoral está determinada principalmente por el estadio y la estirpe, más que por el abordaje quirúrgico. ${ }^{15}$

El objetivo es presentar nuestra experiencia con los primeros 110 casos de nefrectomía laparoscópica realizados en el Centro Médico $\mathrm{ABC}$ en los últimos 14 años.

\section{MATERIAL Y MÉTODOS}

De enero 2006 a enero del 2020 se realizaron 110 nefrectomías laparoscópicas. Todos los pacientes con indicación de nefrectomía fueron considerados como candidatos para abordaje laparoscópico con las si- guientes excepciones: invasión de vena renal o cava, o sospecha de extensión local. Los pacientes candidatos a nefrectomía parcial (tumores sólidos menores de $4 \mathrm{~cm}$, tumores renales bilaterales o en monorrenos) no fueron considerados para nefrectomía radical laparoscópica y se sometieron a nefrectomía parcial abierta, laparoscópica o robótica, excepto en casos aislados de tumores intrarrenales, con involucro de hilio renal o una calificación R.E.N.A.L. mayor a $10 .{ }^{16}$

Se creó una base de datos que incluyó edad, sexo, indicación de cirugía, tiempo quirúrgico, sangrado, complicaciones intraoperatorias, conversiones y complicaciones postoperatorias (incluyendo aquéllas que ocurrieron un mes después de cirugía). También se documentó el resultado histopatológico y el seguimiento del paciente.

Todos los procedimientos se realizaron bajo anestesia general con/sin anestesia regional.

El abordaje quirúrgico fue transabdominal en todos los casos por preferencia del cirujano (Carlos Vázquez Lastra). Se utilizó una técnica de 3-4 puertos, como ya ha sido descrito en otras publicaciones, ${ }^{17}$ o bien, mediante laparoscopia mano asistida en caso de nefrectomía radical para extracción de la pieza quirúrgica a través del puerto de asistencia manual. En dichos casos, la incisión se realizó de 6-7 cm, teniendo que extenderla de acuerdo con el tamaño de la pieza. En caso de nefrectomías simples con riñones atróficos, la pieza se extrajo mediante la ampliación de uno de los puertos laparoscópicos. En los casos de nefroureterectomía, se extrajo la pieza mediante una incisión tipo Gibson modificada, ${ }^{18}$ la cual se utilizó para completar la disección ureteral distal y el rodete vesical.

\section{RESULTADOS}

El número de casos laparoscópicos aumentaron progresivamente durante el periodo de estudio, con 49 del 2006 al 2013 y 61 del 2014 al 2020 . La edad promedio fue de 60 años (rango de 18 a 87 años). La distribución entre ambos sexos fue muy semejante (54 masculinos vs $46 \%$ femeninos). Las características de 
la población estudiada y los datos perioperatorios se incluyen en la Tabla 1.

La indicación de cirugía incluyó cáncer renal en $67(61 \%)$ casos, tumores de urotelio en $22(20 \%)$ y exclusiones renales en 21 casos (19\%) (Tabla 2). En los casos de nefrectomía radical por cáncer renal, el tamaño promedio fue de $6.4 \mathrm{~cm}$, con un rango de 2.7 a $12 \mathrm{~cm}$.

Treinta y siete pacientes (33\%) ya tenían antecedente de cirugía abdominal previa. El tiempo promedio fue de 131 minutos (rango 50 a 300 minutos), la estancia intrahospitalaria fue de 2.7 días (rango 2-7 días). El sangrado promedio fue de $103 \mathrm{~mL}$ (rango 10-2,000 mL). Sólo se requirió transfundir a cuatro pacientes (3\%).

Se registraron complicaciones transoperatorias en tres casos que fueron lesiones vasculares advertidas, las que incluyeron vena renal en uno, arteria renal en uno y lesión de vena adrenal derecha en un caso. En dos pacientes se identificó lesión del bazo, con reparación laparoscópica y aplicación de materiales hemostáticos, sin requerir esplenectomía y sin incremento de volumen de sangrado significativo. Ambos pacientes tuvieron como característica tumores renales mayores a $8 \mathrm{~cm}$ y localizados en polo superior del riñón izquierdo. No hubo conversiones ni mortalidad perioperatoria. Sólo hubo un caso que requirió reintervención por la presencia de sangrado importante, identificado en el postoperatorio inmediato, el cual se resolvió de manera laparoscópica identificando la vena adrenal como el sitio del sangrado y resolviéndolo mediante la aplicación de un clip vascular.

Se observaron complicaciones menores en siete pacientes. Dos presentaron retención urinaria, uno presentó hematoma en uno de los sitios de colocación de trocares que se autolimitó y un paciente presentó hernia postinsicional en uno de los puertos, que requirió posterior resolución quirúrgica. En la Tabla 3 se muestran las complicaciones de acuerdo con la clasificación de Clavien-Duo.

El reporte histopatológico se resume en la Tabla 2. De los 110 casos, 89 (81\%) fueron oncológicos, sien-

Tabla 1: Características de la población estudiada y datos perioperatorios.

\begin{tabular}{lcc}
\hline & Promedio & Rango \\
\hline Edad (años) & 60.0 & $18-87$ \\
Tiempo quirúrgico (min) & 131.0 & $50-300$ \\
Sangrado $(\mathrm{mL})$ & 103.0 & $10-2,000$ \\
Días de estancia intrahospitalaria & 2.7 & $2-7$ \\
\hline
\end{tabular}

Tabla 2: Reporte histopatológico final.

\begin{tabular}{lc}
\hline & $\mathrm{n}(\%)$ \\
\hline Carcinoma renal de células claras & $46(42.0)$ \\
Carcinoma cromófobo de células renales & $6(5.0)$ \\
Carcinoma papilar de células renales & $6(5.0)$ \\
Con componente sarcomatoide & $6(5.0)$ \\
Oncocitoma & $3(3.0)$ \\
Atrofia renal & $21(19.0)$ \\
Carcinoma de células transicionales & $22(20.0)$ \\
\hline
\end{tabular}

Tabla 3: Complicaciones de acuerdo con clasificación Clavien-Duo.

\begin{tabular}{lll}
\hline Clavien & & $\%$ \\
\hline I & 3 & 2.0 \\
II & 4 & 3.0 \\
IIla & 1 & 0.9 \\
IIIb & 2 & 1.8 \\
\hline
\end{tabular}

do el carcinoma de células renales el más frecuente, seguido de carcinoma de células transicionales, sarcoma renal y oncocitoma. En los casos oncológicos, todos se reportaron con márgenes negativos.

El seguimiento de los pacientes consistió en cita a la semana de la cirugía, al mes, a los tres meses y de allí de acuerdo con el seguimiento oncológico establecido para cada caso.

\section{DISCUSIÓN}

La cirugía laparoscópica renal -incluyendo nefrectomía simple, nefrectomía radical, nefrectomía de donador vivo, nefrectomía parcial y nefroureterectomía- sigue siendo uno de los procedimientos laparoscópicos más utilizado por los urólogos. Las últimas décadas han visto un cambio radical en el manejo de cirugía renal. El manejo de tumores renales sólidos con nefrectomía parcial está reemplazando rápidamente a la cirugía radical, y la cirugía robótica para estos casos está reemplazando a la cirugía laparoscópica convencional, lo que deja a la nefrectomía parcial abierta como una alternativa ya en desuso.

En la década de 1990, la urología se encontraba rezagada comparada con cirugía general en la introducción de la laparoscopia en la práctica quirúrgica diaria. Debido parcialmente a la localización retroperitoneal o pélvica de los órganos a tratar, que requería un abordaje más laborioso que el necesario para 
cirugía general, así como un riesgo de sangrado mayor debido al aporte sanguíneo del riñón, pero también por no existir un volumen quirúrgico comparado con colecistectomía o apendicectomía. Es por ello que el urólogo que se inicia en la cirugía laparoscópica tiene que enfrentar un procedimiento de mayor complejidad desde un inicio y sin un volumen adecuado para poder tener una curva de aprendizaje lo suficientemente rápida para disminuir complicaciones.

La primera nefrectomía laparoscópica realizada en un humano por Clayman en 1990 fue un procedimiento sin complicaciones, de 8 horas de duración y con el reporte final de un oncocitoma de $4 \mathrm{~cm}$. A partir de ese momento histórico, el interés del mundo urológico por la cirugía laparoscópica ha crecido de forma exponencial. Este interés se vería reflejado en el advenimiento de la cirugía robótica en 1997, donde después de realizarse la primera colecistectomía robótica, la plataforma de cirugía robótica encontró en la urología la especialidad que la impulsaría a ser lo que es hoy en día.

La nefrectomía laparoscópica ha demostrado ser un procedimiento seguro con ventajas significativas sobre la cirugía abierta. Los procedimientos laparoscópicos han demostrado menor morbilidad, menos sangrado, al igual que requerimiento de analgésicos y reintegración más rápida a actividades normales, sin comprometer los resultados oncológicos. Por ello, la tendencia mundial ha sido a realizar estos procedimientos de forma laparoscópica, dejando los procedimientos abiertos para ciertas indicaciones particulares, principalmente referente al estadio, tamaño y extensión local del tumor. La calidad de vida postoperatoria, definida como la combinación de diferentes variables (dolor postoperatorio, tiempo de hospitalización, periodo de convalecencia y tiempo a recuperación completa) ha demostrado ser superior en nefrectomía laparoscópica comparada con nefrectomía abierta. ${ }^{19}$

El tiempo promedio de regreso a actividad normal ha sido reportado de 3.6 semanas en el grupo laparoscópico, comparado con 8.1 semanas en la cirugía abierta, ${ }^{20}$ y el tiempo promedio para recuperación total de 8.2 semanas comparado con 29.3 semanas, respectivamente. ${ }^{21}$

Los reportes de control oncológico a cinco, 10 y 15 años no han mostrado inferioridad del abordaje laparoscópico, en comparación con el abordaje abierto. ${ }^{22,23}$

Las indicaciones de nefrectomía laparoscópica son esencialmente las mismas que para nefrectomía abierta, excepto en casos de tumores muy volumino- sos, sospecha de involucro de vena renal y de involucro perirrenal importante por proceso inflamatorio, como sucede en los casos de pielonefritis xantogranulomatosa. En nuestro estudio, éstos fueron criterios de exclusión para no considerar un procedimiento laparoscópico. En cuanto al tamaño del tumor, hay controversia respecto al diámetro mayor capaz de ser removido de forma laparoscópica. Inicialmente se había creado un límite de $10 \mathrm{~cm}$; sin embargo, conforme este procedimiento se ha vuelto una herramienta cotidiana para el urólogo, y conforme aumenta su curva de experiencia, es posible realizar nefrectomía de tumores de mayor tamaño. ${ }^{24}$

La complicación transoperatoria observada en nuestra serie fue lesión vascular (3 casos, $2 \%$ ). No obstante, todas se pudieron resolver de forma laparoscópica, no requirieron necesidad de convertir ninguno de los casos de esta serie. Estos pacientes no se encontraban en el percentil de tumores de mayor tamaño, o fue particular de cirugía oncológica o nefrectomía simple, por lo tanto, concluimos que aunque el tamaño tumoral representa un factor que puede requerir de mayor experiencia del cirujano, la nefrectomía laparoscópica es un procedimiento que puede presentar complicaciones vasculares para las que se debe estar preparado. Las lesiones vasculares siguen siendo las complicaciones más frecuentes en este procedimiento. ${ }^{25}$

Desde el punto de vista técnico, hay dos formas de abordar la cirugía laparoscópica renal: el abordaje transabdominal y el retroperitoneal. Los estudios comparativos no han demostrado superioridad de uno sobre el otro, por lo que el abordaje sigue siendo una decisión de acuerdo con la preferencia del cirujano. ${ }^{26,27}$ En el abordaje transabdominal, el espacio de trabajo es mayor y la orientación más fácil, aunque llegar al hilio renal y realizar la disección renal puede volverse más laborioso. En el abordaje retroperitoneal, el riñón y los vasos renales son relativamente más fáciles de abordar; sin embargo, el espacio de trabajo es menor, la orientación es más difícil y la ergonomía del procedimiento no es óptima. Una de las limitantes del procedimiento transabdominal es el antecedente de cirugías abdominales previas. En esta serie, $33 \%$ de los pacientes habían tenido alguna cirugía transabdominal previa. Aunque algunos de estos casos sí requirieron tiempo quirúrgico en liberación de adherencias, no se reportó la imposibilidad de realizar el procedimiento por este antecedente. Otras publicaciones han demostrado también la seguridad del abordaje transabdominal con cirugías abdominales previas. ${ }^{28}$ 
El tiempo quirúrgico promedio fue 131 minutos (rango de 50 a 300), y observamos una disminución en éste durante la serie. Al comparar el tiempo quirúrgico de la primera mitad de casos (141 minutos) con el de la segunda mitad (118 minutos), notamos menor tiempo quirúrgico, relacionado con la experiencia y la estandarización del procedimiento.

La seguridad oncológica de la nefrectomía laparoscópica ya ha sido demostrada por diferentes autores. ${ }^{29,30}$

El seguimiento a largo plazo de la nefrectomía laparoscópica confiere los mismos resultados que la cirugía abierta.

\section{CONCLUSIONES}

Considerando la información existente, así como nuestra experiencia personal, la nefrectomía laparoscópica se puede considerar un procedimiento de rutina, seguro y efectivo, asociado con mínima morbilidad con ventajas significativas para el paciente. En la actualidad, se ha establecido como el estándar de oro para el manejo de la patología oncológica y benigna renal. Ya sea mediante laparoscopia pura o mano asistida, el resultado estético, el requerimiento de analgésicos y la velocidad para reintegrar al paciente a sus actividades diarias coloca a esta técnica por arriba de la cirugía abierta. Con tiempos quirúrgicos semejantes e incluso menores que la cirugía abierta, la cirugía laparoscópica es el método de elección hoy en día para aquellos pacientes bien seleccionados, una vez descartada la posibilidad de realizar una cirugía preservadora de nefronas.

\section{REFERENCIAS}

1. Clayman RV, Kavoussi LR, Soper NJ, Dierks SM, Meretyk S, Darcy MD et al. Laparoscopic nephrectomy: initial case report. J Urol. 1991; 146 (2): 278-282.

2. Eskicorapci SY, Teber D, Schulze M, Ates M, Stock C, Rassweiler JJ. Laparoscopic radical nephrectomy: the new gold standard surgical treatment for localized renal cell carcinoma. ScientificWorldJournal. 2007; 7: 825-836. doi: 10.1100/tsw.2007.153.

3. Raghuram S, Godbole HC, Dasgupta P. Laparoscopic nephrectomy: the new gold standard? Int J Clin Pract. 2005; 59 (2): 128-129. doi: 10.1111/j.1742-1241.2005.00470.x.

4. Peña González JA, Pascual Queralt M, Salvador Bayarri JT, Rosales Bordes A, Palou Redorta J, Villavicencio Mavrich H. Evolución de la cirugía abierta versus laparoscópica/robótica: 10 años de cambios en Urología. Actas Urol Esp. 2010; 34 (3): 223-231.

5. Inman BA. Open versus laparoscopic nephroureterectomy: is there really a debate? Eur Urol. 2012; 61 (4): 722-724. doi: 10.1016/j.eururo.2011.12.051.
6. Kavoussi LR, Kerbl K, Capelouto CC, McDougall EM, Clayman RV. Laparoscopic nephrectomy for renal neoplasms. Urology. 1993; 42 (5): 603-609.

7. Kutikov A, Smaldone MC, Egleston BL, Uzzo RG. Should partial nephrectomy be offered to all patients whenever technically feasible? Eur Urol. 2012; 61 (4): 732-734; discussion 734-735.

8. Anele UA, Marchioni M, Yang B, Simone G, Uzzo RG, Lau C et al. Robotic versus laparoscopic radical nephrectomy: a large multi-institutional analysis (ROSULA Collaborative Group). World J Urol. 2019; 37 (11): 2439-2450. doi: 10.1007/s00345019-02657-2.

9. Alimi Q, Peyronnet B, Sebe P, Cote JF, Kammerer-Jacquet $\mathrm{SF}$, Khene ZE et al. Comparison of short-term functional, oncological, and perioperative outcomes between laparoscopic and robotic partial nephrectomy beyond the learning curve. J Laparoendosc Adv Surg Tech A. 2018; 28 (9): 1047-1052. doi: 10.1089/lap.2017.0724.

10. Carrión DM, Gómez Rivas J, Aguilera Bazán A, Alonso y Gregorio S, De Castro Guerín C, Álvarez-Maestro M et al. Laparoscopic donor nephrectomy versus open donor nephrectomy: Outcomes from a single transplant center. Arch Esp Urol. 2019; 72 (5): 508-514.

11. Wilson CH, Sanni A, Rix DA, Soomro NA. Laparoscopic versus open nephrectomy for live kidney donors. Cochrane Database Syst Rev. 2011; (11): CD006124. doi: 10.1002/14651858. CD006124.pub2.

12. Nouralizadeh A, Tabatabaei S, Basiri A, Simforoosh N, Soleimani M, Javanmard B et al. Comparison of open versus laparoscopic versus hand-assisted laparoscopic nephroureterectomy: a systematic review and meta-analysis. J Laparoendosc Adv Surg Tech A. 2018; 28 (6): 656-681. doi: 10.1089/lap.2017.0662.

13. Peyronnet B, Seisen T, Dominguez-Escrig JL, Bruins HM, Yuan CY, Lam T et al. Oncological outcomes of laparoscopic nephroureterectomy versus open radical nephroureterectomy for upper tract urothelial carcinoma: an European Association of Urology Guidelines Systematic Review. Eur Urol Focus. 2019; 5 (2): 205-223. doi: 10.1016/j. euf.2017.10.003.

14. Liu G, Ma Y, Wang S, Han X, Gao D. Laparoscopic versus open radical nephrectomy for renal cell carcinoma: a systematic review and meta-analysis. Transl Oncol. 2017; 10 (4): 501 510. doi: 10.1016/j.tranon.2017.03.004.

15. Thompson RH, Hill JR, Babayev Y, Cronin A, Kaag M, Kundu $\mathrm{S}$ et al. Metastatic renal cell carcinoma risk according to tumor size. J Urol. 2009; 182 (1): 41-45.

16. Kutikov A, Uzzo RG. The R.E.N.A.L. nephrometry score: a comprehensive standardized system for quantitating renal tumor size, location and depth. J Urol. 2009; 182 (3): 844-853. doi: 10.1016/j.juro.2009.05.035.

17. Bove P, Iacovelli V, Sandri M, Carilli M, Cindolo L, Autorino $\mathrm{R}$ et al. Entry techniques in laparoscopic radical and partial nephrectomy: a multicenter international survey of contemporary practices. Minerva Urol Nefrol. 2018; 70 (4): 414-421. doi: 10.23736/S0393-2249.18.03075-8.

18. Yang WH, Ou CH. A muscle-sparing modified Gibson incision for hand-assisted retroperitoneoscopic nephroureterectomy and bladder cuff excision--an approach through a window behind the rectus abdominis muscle. Urology. 2012; 79 (2): 470-474. doi: 10.1016/j. urology.2011.09.043.

19. Acar C, Bilen C, Bayazit Y, Aslan G, Koni A, Basok E et al. Quality of life survey following laparoscopic and open radical nephrectomy. Urol J. 2014; 11 (6): 1944-1950. 
20. Bahler CD, Sundaram CP. Quality of life following laparoscopic living-donor nephrectomy. JSLS. 2013; 17 (2): 273-278. doi: 10 .4293/10860813X13693422521395.

21. Parker PA, Swartz R, Fellman B, Urbauer D, Li Y, Pisters LL et al. Comprehensive assessment of quality of life and psychosocial adjustment in patients with renal tumors undergoing open, laparoscopic and nephron sparing surgery. J Urol. 2012; 187 (3): 822-826. doi: 10.1016/j.juro.2011.10.151.

22. Shigeta K, Kikuchi E, Abe T, Hagiwara M, Ogihara K, Anno $\mathrm{T}$ et al. Long-term oncologic outcomes of laparoscopic versus open radical nephroureterectomy for patients with T3N0M0 upper tract urothelial carcinoma: a multicenter cohort study with adjustment by propensity score matching. Ann Surg Oncol. 2019; 26 (11): 3774-3781. doi: 10.1245/s10434-01907623-1.

23. Colombo JR Jr, Haber GP, Jelovsek JE, Lane B, Novick AC, Gill IS. Seven years after laparoscopic radical nephrectomy: oncologic and renal functional outcomes. Urology. 2008; 71 (6): 1149-1154. doi: 10.1016/j.urology.2007.11.081.

24. Kruck S, Anastasiadis AG, Walcher U, Stenzl A, Herrmann TR, Nagele U. Laparoscopic partial nephrectomy: risk stratification according to patient and tumor characteristics. World J Urol. 2012; 30 (5): 639-646. doi: 10.1007/s00345-0110806-9.

25. Gill IS, Kavoussi LR, Clayman RV, Ehrlich R, Evans R, Fuchs $\mathrm{G}$ et al. Complications of laparoscopic nephrectomy in 185 patients: a multi-institutional review. J Urol. 1995; 154 (2 Pt 1): 479-483. doi: 10.1097/00005392-199508000-00037.

26. Taue R, Izaki H, Koizumi T, Kishimoto T, Oka N, Fukumori $\mathrm{T}$ et al. Transperitoneal versus retroperitoneal laparoscopic radical nephrectomy: a comparative study. Int J Urol. 2009; 16 (3): 263-267. doi: 10.1111/j.1442-2042.2008.02219.x.

27. Savran-Karadeniz M, Kisa I, Salviz EA, Ozkan-Seyhan T, Tefik $\mathrm{T}$, Sanli $\mathrm{O}$ et al. Can surgical approach affect postoperative analgesic requirements following laparoscopic nephrectomy: Transperitoneal versus retroperitoneal? A prospective clinical study. Arch Esp Urol. 2017; 70 (6): 603-611.

28. Yanai Y, Takeda T, Miyajima A, Matsumoto K, Hagiwara $\mathrm{M}$, Mizuno $\mathrm{R}$ et al. Is transperitoneal laparoscopic radical nephrectomy suitable for patients with a history of abdominal surgery? Asian J Endosc Surg. 2019; 12 (4): 429-433. doi: 10.1111/ases.12673.

29. Tait C, Tandon S, Baker L, Goodman C, Townell N, Nabi G. Long-term oncologic outcomes of laparoscopic radical nephrectomy for kidney cancer resection: Dundee cohort and metaanalysis of observational studies. Surg Endosc. 2011; 25 (10): 3154-3161. doi: 10.1007/s00464-011-1736-8.

30. Laird A, Stewart GD, Zhong J, Ang WJ, Cutress ML, Riddick $\mathrm{AC}$ et al. A generation of laparoscopic nephrectomy: stagespecific surgical and oncologic outcomes for laparoscopic nephrectomy in a single center. J Endourol. 2013; 27 (8): 1008-1014. doi: 10.1089/end.2012.0562. 